\title{
FEASIBILITY STUDY OF LOW-COST IMAGE-BASED HERITAGE DOCUMENTATION IN NEPAL
}

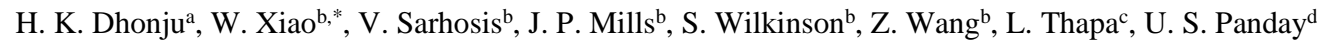

\author{
${ }^{a}$ International Centre for Integrated Mountain Development (ICIMOD), Kathmandu, Nepal - hari.dhonju@icimod.org \\ b School of Civil Engineering and Geosciences, Newcastle University, NE1 7RU, Newcastle upon Tyne, UK - wen.xiao@ ncl.ac.uk \\ ${ }^{\mathrm{c}}$ Cadastral Survey Division, Survey Department, Ministry of Land Reform and Management, Nepal \\ ${ }^{\mathrm{d}}$ Department of Civil and Geomatics Engineering, Kathmandu University, Nepal
}

\section{Commission II}

KEY WORDS: 3D modelling, Crowd-source, Heritage documentation, Low-cost, Photogrammetry

\begin{abstract}
:
Cultural heritage structural documentation is of great importance in terms of historical preservation, tourism, educational and spiritual values. Cultural heritage across the world, and in Nepal in particular, is at risk from various natural hazards (e.g. earthquakes, flooding, rainfall etc), poor maintenance and preservation, and even human destruction. This paper evaluates the feasibility of low-cost photogrammetric modelling cultural heritage sites, and explores the practicality of using photogrammetry in Nepal. The full pipeline of 3D modelling for heritage documentation and conservation, including visualisation, reconstruction, and structure analysis, is proposed. In addition, crowdsourcing is discussed as a method of data collection of growing prominence.
\end{abstract}

\section{INTRODUCTION}

Located on a ridge of the Tibetan and Indian Plates, Nepal is extremely prone and sensitive to natural disasters. Among the most devastating of events, Nepal experienced a 7.8 magnitude earthquake on 25 April, 2015. The impact of the earthquake was extensive on historically and culturally important heritage sites that have great spiritual and religious value. According to Nepal's Department of Archaeology, around 750 heritage structures, e.g. temples and shrines, of significant culture and religious value were affected. Many heritage structures were already in danger, e.g. Kathmandu Valley was once listed on the UNESCO List of World Heritage in Danger, and became more vulnerable after this devastating earthquake. Some sites were totally destroyed, and some were partially damaged and are likely to collapse in the future. Critically, remaining intact heritage that survived now needs to be protected and preserved.

The importance of cultural and historical heritage structural documentation is gaining momentum and is well recognized internationally (Remondino and Rizzi, 2010). There is an imperative need and responsibility to protect heritage sites and conserve cultural and religious values for future generations. There is increasing demand to document and preserve heritage digitally for later stage usage such as visualization. The continuous development of new sensors, data capture capability, 3D modelling technologies, and online visualization can contribute significantly to $3 \mathrm{D}$ documentation, conservation, and digital presentation of heritage structures, which attracts growing research interest in this field (Remondino, 2011).

Over recent years, it has become increasingly common to use digitization and 3D modelling for preservation and conservation of heritage sites due to advances in lidar (light detection and ranging)-based and image-based modelling and visualization techniques towards virtual reality. 3D construction methods based on laser scanning and automated image-based techniques are both widely applied to heritage documentation. In many cases, it is best to take advantage of both techniques by fusing different data sources. However, given the fact that expensive laser scanning equipment may not be available in developing countries, this paper studies the feasibility of a low-cost, easy to use, and high quality image-based surveying techniques



Figure 1. Heritage temples in Kathmandu Durbar Square.

accompanied with open-source 3D reconstruction methods for the documentation of complex heritage structures.

Kathmandu Valley is characterised by rich ancient inscriptions, sculptures and monuments of various size and shapes, and was listed as a UNESCO World Heritage Site in 1979. Figure 1 shows heritage temples under poor maintenance harmed by the earthquake in the centre of Kathmandu Durbar Square. In line with uncontrolled urbanization and loss of historic fabric, the world heritage sites in Kathmandu Valley were placed on the danger list in 2003. To control this trend, an integrated management plan (IMP) was introduced by the Government of Nepal and the sites were withdrawn from the danger list in 2007 (Acharya and Pradhananga, 2013). Implementation of the IMP was less effective due to difficulties faced in restructuring the institutions for restoration and reconstruction processes. Traditionally, communities actively participated in conservation, preservation and restoration of heritage affairs (Shakya et al., 2013; Tiwari, 2013). Vulnerability and risks to heritage structures are rapidly growing due to uncontrolled urban expansion, as well as seismic vulnerability and hazards. This leads to the need to assess for disaster risk reduction by mainstreaming cultural heritage for disaster management and the development of tools through the use of appropriate recent technology (Jigyasu, 2013; Maskey, 2013). 


\section{RELATED WORK}

Recent capability of technological advances in documentation have demonstrated the potential for $3 \mathrm{D}$ registration and reconstruction of archaeological and cultural heritage monuments (Pavlidis et al., 2007). The techniques can be broadly categorised into: i) photogrammetry, close-range and imagebased modelling (Hanke and Grussenmeyer, 2002; Remondino and El-Hakim, 2006; Remondino and Menna, 2008; Santagati et al., 2013), and ii) laser scanning or lidar-based modelling (Guarnieri et al., 2004; Mills and Barber, 2004). Unmanned aerial vehicle (UAV) platform has been used for photogrammetric 3D modelling (Remondino, 2011; Eisenbeiss, 2004; Nex and Remondino, 2014), and its usage for laser scanning is still under rapid development. However, laser scanning technique is not trivial and requires a certain degree of expertise and skill while performing archaeological and heritage field work (Reu et al., 2013). Moreover, the technique is often not cost-effective due to the expensive equipment and its time consuming nature.

Numerous literature has discussed different 3D techniques for low cost 3D documentation of cultural heritage (Remondino, 2011; Boochs et al., 2007; Guidi et al., 2007; Kersten and Lindstaedt, 2012; Reu et al., 2013). Mills et al. (2000), for example, investigated early low-cost PC software against stateof-practice photogrammetric instrumentation. More recently, Reu et al. (2013) proposed a 3D cost-effective registration of archaeological heritage. They used the software package PhotoScan, (Professional Edition) which allows the extraction of $3 \mathrm{D}$ point clouds from ordinary $2 \mathrm{D}$ images using the structurefrom-motion (SfM) approach and dense stereo-matching. They claim to be cost effective compared to traditional methods and to provide a fully automated system with high accuracy and simple 3D model generation. However, costs are incurred for acquiring software licenses and high technical skills and knowledge are necessary. Similarly, Boochs et al. (2007) and Kersten and Lindstaedt (2012) demonstrated free or low cost 3D reconstruction methods for archaeological and heritage objects with the combined used of photogrammetric and computer vision techniques. The method is targeted particularly at non-technical users with a different level of geometric accuracy.

In essence, documentation is a necessary step and important within the scope of conservation, preservation and restoration of archaeological and cultural heritage structures (Boochs et al., 2007). And, the heritage sites in the Kathmandu Valley are very specific in terms of complexity and uniqueness of the ancient structures (Kersten and Lindstaedt, 2012) and their vulnerable spatial locations. In the context of Nepal, a feasibility study is proposed using low-cost 3D image-based modelling for heritage conservation with the aim to safeguard heritage in Kathmandu Valley.

\section{PHOTOGRAMMETRIC DOCUMENTATION}

Generally, for different applications, one needs to address different aspects of reconstruction, namely, high geometric accuracy, comprehensive detail capture, photorealism, high automation level, low cost, portability, application flexibility, and model size efficiency (El-Hakim et al., 2004). The priority and importance of these specifications depends on the purpose of 3D modelling, for instance, whether to restore the geometry of the heritage structure for future regeneration or to document for virtual reality targeted at tourism. For the former purpose, accurate dimension and detail carving information of the structure is important and mandatory, whereas for the later textures and photo-realistic details are more important than the accurate dimensions of the model.

In this study, the importance of documentation and its specification for actual 3D image-based surveying, modelling techniques and methodologies, including their limitations and potential, will be discussed. Examples of UNESCO world heritage structure 3D reconstructions are presented and discussed.

\subsection{Photogrammetric modelling for heritage conservation}

The photogrammetric reconstruction procedure, i.e. SfM, has been validated in many applications, including heritage documentation. Figure 2 illustrates the diagram of photogrammetric 3D reconstruction for heritage conservation.

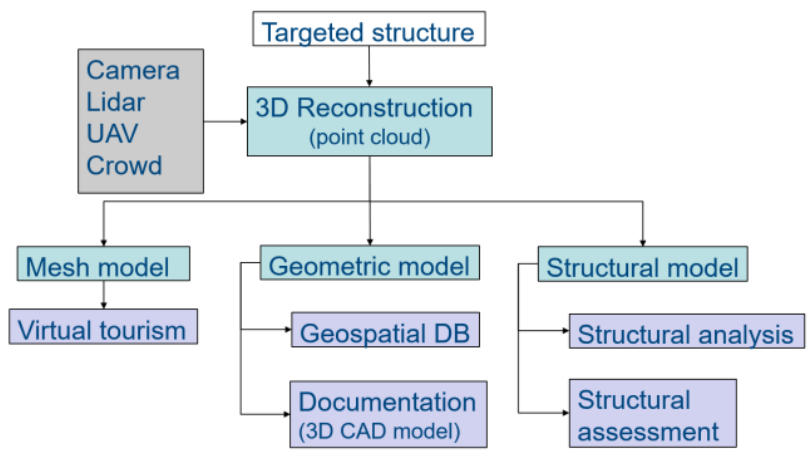

Figure 2. Diagram of photogrammetric reconstruction workflow for heritage structure conservation.

Input: For photogrammetric 3D modelling, optical images from cameras on different platforms are the primary source of data input. High resolution, well-calibrated surveying cameras will produce high quality images, and hence better 3D models are expected. However, low-cost consumer grade cameras, or even mobile phone cameras can yield useful models, and the use of them for heritage documentation is worthy of further investigation.

Besides photographic data, laser scanning data have long been used for accurate 3D modelling, e.g. building information modelling (BIM). There is an entire industrial pipeline for rangebased modelling, from data acquisition, to registration, to (semi)automatic vectorization, and finally presentation. The use of images to complement laser scanning data, which is often purely geometric, is also well studied. However, the limitation is that a laser scanner is often very expensive. Therefore, it is not widely used in many developing countries such as Nepal. This paper will therefore focus solely on image-based modelling.

Ground-based image/laser scanning data acquisition has limited points of view, for instance building rooftops cannot be reached, hence one usually cannot obtain full coverage of an object of interest. One popular technique is therefore the use of UAVs, which have very flexible acquisition perspectives and can attain a large coverage with varied spatial resolution. The concern of using UAVs is security and safety issues, due to which flying permission can be difficult to obtain.

In recent years, crowd-sourced information collection and processing has also become popular. Crowd-sourced photogrammetric 3D reconstructions can offer unique opportunities for the digital interactive visualization of lost 
heritage (Vincent et al., 2015). This technique provides a platform to preserve and revive lost heritage in order to recall the memory of that heritage through digital preservation schemes. Even though true geometry of heritage is difficult to recover using crowd-sourced data, it can still help to address community interests. At the end, the 3D textured and realistic model can add value to visualizations, remembrance and digital documentation.

Output: The primary product of photogrammetric reconstruction is a group of points, usually called a point cloud, representing the shape of the structure. Based on this point cloud, other data formats of the structure can be generated, such as mesh models, geometric models, and structural models (detailed construction elements of an object). These models can be used for different aspects of heritage conservation.

Models: The most common product of photogrammetric 3D modelling is a mesh model, which is triangulated from the original point cloud. As this kind of mesh model is textured and photorealistic, it is widely used for visualization purposes. As for heritage conservation, it can be used in online virtual tourism to attract tourists. Mesh models can also be transferred to printable format, such as STL files, for 3D printing.

The other usage of the photogrammetric point cloud data is to generate geometric models by vectorization, such AutoCAD and CityGML models. These models maintain accurate spatial and geometric features, and thus can be integrated into geospatial databases for better urban planning and design, and preserved for documentation and reconstruction purposes. Some heritage structures may have designed CAD models, but new models based on real world data acquisition can validate and update the current models, and can be of even greater culture value.

Based on geometric models, an even more detailed structural model capturing the exact geometry of individual bricks and mortar in a historic masonry structure can be constructed. Such structural model can then be coupled with structural engineering tools, such as advanced 3D finite and discrete element methods of analysis (Bui et al., 2017; Giamundo et al., 2014), to assess the robustness and vulnerability of historic structures to extreme natural disasters, e.g. earthquakes, flooding (Sarhosis et al., 2016). In addition, advanced algorithms for automatic detection of structural damages and material degradation in the structure can be developed to assist with the structural health monitoring and assessment of historic structures. Such tools can be extremely useful to the structural engineers since accurate information about the current state of the structure could be obtained and predictions about the long-term behaviour of the structure subjected to various loading conditions can be assed.

\subsection{Consumer-grade cameras for 3D modelling}

To assess the feasibility of low-cost image-based reconstruction methods for Nepalese heritage structures, images acquired from a DSLR camera and a mobile phone camera (Table 1) were used for 3D reconstruction using commercial software, Bentley's ContextCapture (former Acute $3 \mathrm{D}^{1}$ ). Preliminary tests using an open source 3D reconstruction package, VisualSfM, generated recognisable, but still far from usable, structures. Both DSLR (Figure 3) and phone (Figure 4) cameras can generate visually appealing 3D models, especially when the structure is relatively small. Detailed information can be extracted from these imagebased reconstructions.

\footnotetext{
${ }^{1}$ www.acute3d.com
}

Table 1. Utilised DSLR and mobile phone cameras.

\begin{tabular}{|l|c|c|}
\hline Camera & \multicolumn{2}{|c|}{ Specifications } \\
\hline & Sensor size & Focal length \\
Canon EOS 600D & $22.3 \mathrm{~mm}$ & $18 \mathrm{~mm}$ \\
Huawei Nexus 6P & $6.2 \mathrm{~mm}$ & $4.67 \mathrm{~mm}$ \\
\hline
\end{tabular}

Figure 3 illustrates a small temple in Patan Durbar Square, Kathamndu, and Figure 4 shows a mini statue on a wall of a temple in Bhaktapur. More models in 3D can be found on the project website ${ }^{2}$.

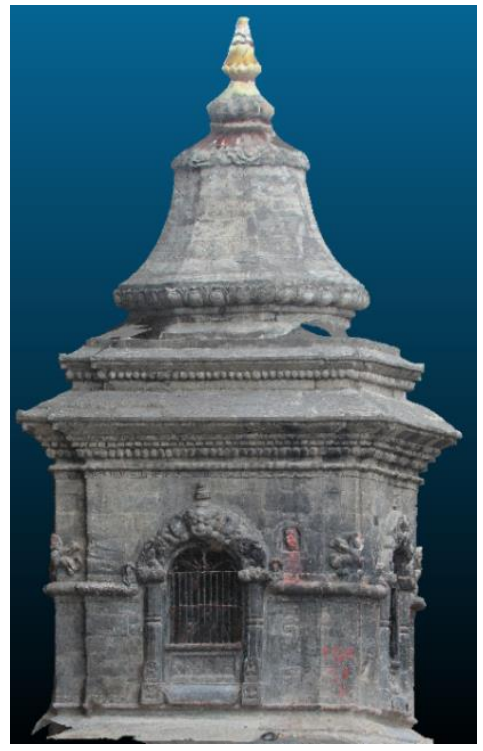

Figure 3. 3D model of a small temple in Patan Durbar Square.



Figure 4. 3D model of a mini statue of a temple in Bhaktapur.

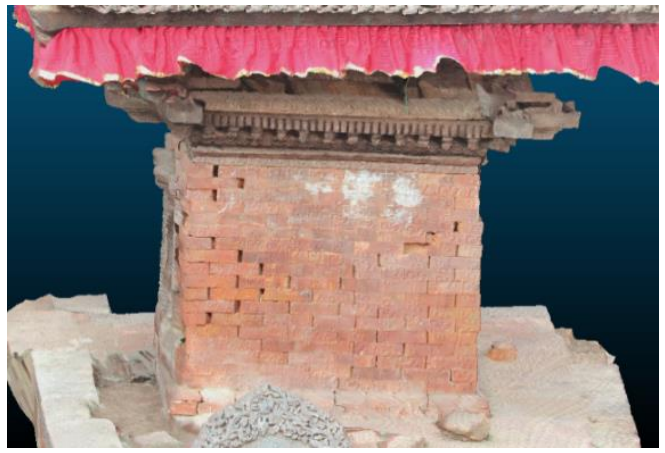

Figure 5. Model of a temple with cracks induced by earthquake.

${ }^{2}$ http://research.ncl.ac.uk/heritageconservation/ 
As mentioned, the original point cloud can be transferred into different model formats for various applications. For instance, structural damage induced cracks can be captured by the 3D model (Figure 5), showing the potential of using this technique for structural assessment and analysis.

One of the reasons to assess the usability of low-cost 3D modelling, such as using phone cameras, is that non-experts (ordinary people with only limited knowledge of photogrammetric $3 \mathrm{D}$ reconstruction) can contribute to heritage preservation and documentation. Images of heritage sites taken by tourists can be used for 3D reconstruction. Moreover, civilians can use their phones to take images to help document their nation's culture treasures. Figure 6 shows an endangered monument, awaiting demolition, which is covered by scaffolding. In its present state it is no longer possible to document the structure as it has mostly been obscured. However, images taken prior to the erection of the scaffolding by local people have been used to generate a useful 3D model.



Figure 6. A monument covered by scaffolds and its 3D model produced using crowdsourced data before scaffolding.

With the ubiquity of mobile phones, crowdsourcing has been an important method of data collection, and has been successfully applied for 3D modelling. There are currently a number of research projects related to crowdsourcing for heritage conservation, such as Curious Travellers ${ }^{3}$ and Project Mosul ${ }^{4}$. These projects encourage volunteers to upload their images of heritage sites, however often do not have any specifications for the photographs, such as a requirement for geotags.

\subsection{Practical limitations of image-based documentation}

There are clear advantages of image-based documentation, such as photorealism, portability, application flexibility, and low cost, making it an obvious choice. However, there are also other aspects to consider in order to achieve high quality modelling, for example geometric accuracy, level of detail, completeness, automation level, and model size efficiency. Photogrammetric modelling is useful in certain situations, but there are practical limitations.

Firstly, light condition plays an important role in image acquisition. It is better to have bright and clear images to produce a higher quality model. In some cases, artificial lighting might be

\footnotetext{
${ }^{3}$ www.visualisingheritage.org
}

needed to capture ideal images. Photogrammetric 3D modelling relies on representative common pixels (corresponding feature points) from different images taken from various perspectives to reconstruct the camera locations and geometry of the object. Therefore, it might fail on structures on which there are repetitive patterns. It is better to capture the salient part of the object, and artificial targets can be used to strengthen the image matching. In addition, imaging angles can also influence the modelling result, as camera lenses will generate image distortions, especially lowcost ones, even if it can be rectified during the reconstruction process. It is recommended to take orthogonal photos, meaning having a vertical viewing angle. Finally, accessibility is a major constraint for image acquisition. For example, to model a tall and large building, it is always difficult to take images from ideal locations and angles. In dense urban environments, it is common to only have limited access to buildings.

The architecture of many Nepali heritage sites differs from others in terms of complexity and uniqueness of structure. There are usually many hand-carved sculptures for both decoration and support of upper levels, as shown in Figure 7. Such structures can create challenges for image-based reconstruction. Their complexity makes them difficult to model and they can be difficult to access. Moreover, light conditions can be poor as the sculptures are often shadowed by the roof.

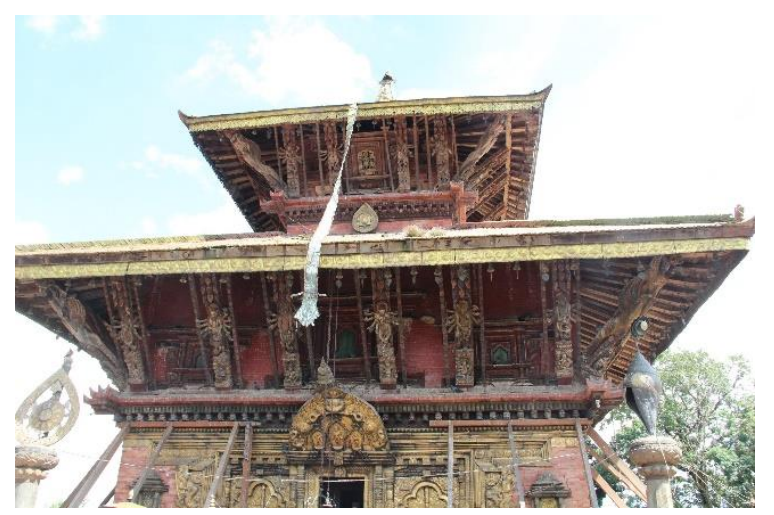

Figure 7. Changu Narayan temple imaged from the ground.

Image-based reconstruction produces decent results at the foot of this type of structure, but sculptures such as supporting pillars are difficult to recover, as are sections shadowed by the roof. Figure 8 illustrates this problem, where structures beneath the roof are incomplete and badly reconstructed. Besides the challenges of complex structures, the $3 \mathrm{D}$ modelling process also introduces errors and noise due to incorrect dense image matching.

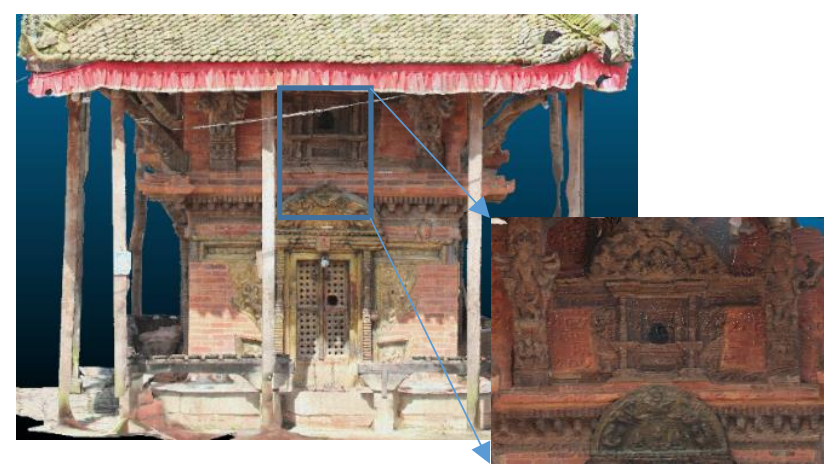

Figure 8. 3D model of a typical Hindu temple in Nepal. Areas covered by the roof are poorly reconstructed.

\footnotetext{
${ }^{4}$ https://projectmosul.org
} 


\section{COMPARISON AND EVALUATION}

To evaluate the geometric quality of low-cost image-based 3D reconstruction, the phone camera (shown in Table 1) and a high precision laser scanner (Leica HDS P20) were used to scan the same building and the two different point clouds were registered and compared. As laser scanning is not available on site in Nepal, an historic building in the UK was taken as an example (Figure 10). 48 images were taken and used for the $3 \mathrm{D}$ modelling. Six stations were planned for the laser scanning, and artificial targets were used to accurate register the scans to form a complete point cloud. The two different point clouds were co-registered manually by CloudCompare ${ }^{5}$.

One thing to note is that the image-based models are not scaled, meaning the model does not reflect the real world structure size. However, this can be achieved by assigning one single scale factor, which can be easily obtained by tape measurement. For the comparison, the scale is approximated during the coregistration procedure while minimising the point-to-point distances using iterative closed point (ICP) between the two point clouds. Then, the point-to-point distances reflect the consistency between these two modelling techniques. Beside the point-topoint distance, the distance between a target point to a nearest local surface in the reference point cloud can also be used. This surface can be a least square plan (fitted by either certain number of points or fixed radius), a triangular or a quadric surface. Only the point-to-point distance comparison is presented in Figure 10.

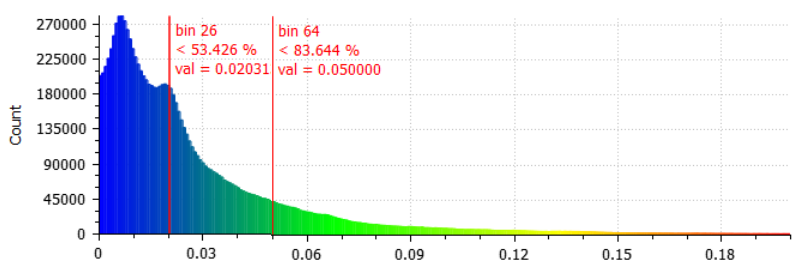

Figure 9: Histogram of distance between two point clouds.

The comparison demonstrates that the photogrammetric method using a phone camera generates a comparable point cloud to that from laser scanning, as more than half of the distances $(53.4 \%)$ were under $2 \mathrm{~cm}$ and the majority of the distances (83.6\%) were smaller than $5 \mathrm{~cm}$ (Figure 9). Here, image distortion was not rectified, and the laser scanned point cloud was simply treated as ground truth, meaning the errors in laser scan registration were not taken into consideration. This accuracy should suffice for the majority of modelling purposes, e.g. documentation, visualisation, and wire-frame model generation.

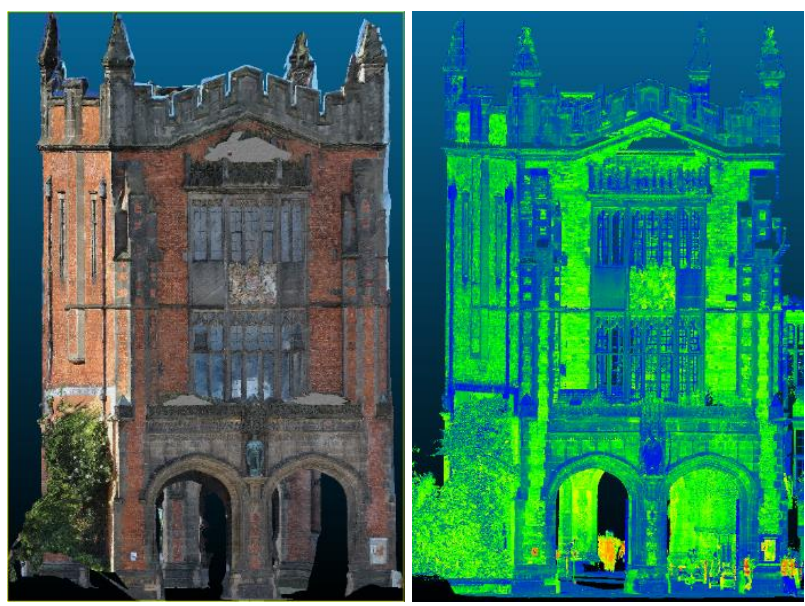

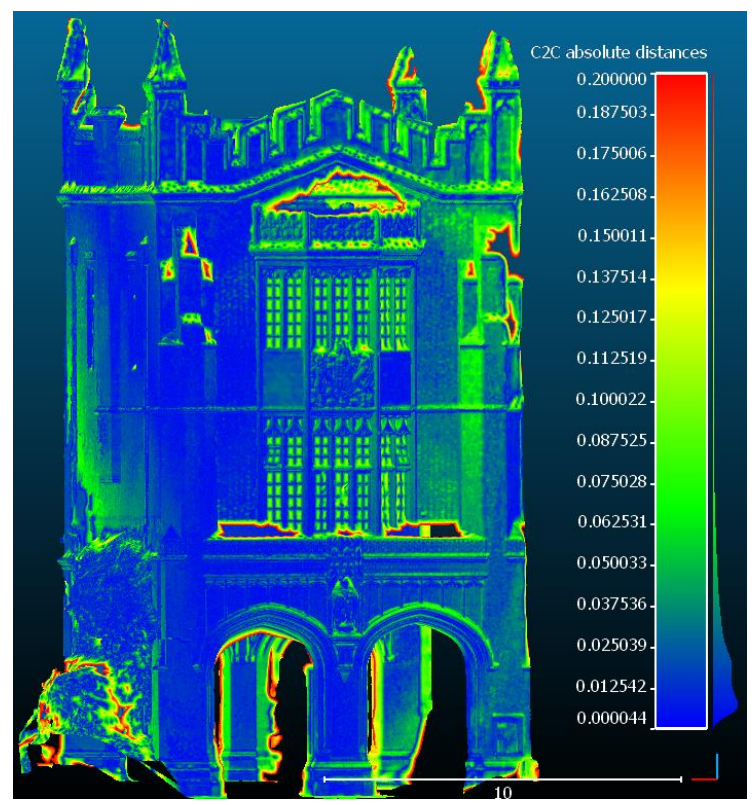

Figure 10. Comparison of image-based 3D modelling point cloud (top left) with laser scanned point cloud (top right). Data were registered manually and compared using CloudCompare ${ }^{5}$.

\section{DICUSSION AND CONCLUSION}

Photogrammetric 3D modelling for heritage documentation is a well-studied topic. This paper has studied the feasibility of lowcost $3 \mathrm{D}$ reconstruction methods for the purpose of heritage conservation in developing countries such as Nepal. Ideally, all data sources, including ground-based cameras, UAVs, laser scanning and crowdsourcing, should be investigated and used for heritage documentation. Range and image-based modelling methods have both pros and cons, and can be complementary to each other.

In general, simple image acquisition is capable of generating point clouds that are comparable to laser scanning. However, due to the often complex structure and shape of cultural heritage objects, a more sophisticated and well planned image acquisition scheme is recommended. Low-cost image-based documentation is still of great value in terms of visualisation of simple models.

Even very simple 2D images can be useful for documentation purposes. Geotagged images can help to identify the accurate number and the structural health and vulnerability of heritage structures. There are thousands of heritage structures that are in danger and in need of special attention and consideration to prolong their lives, especially in remote areas of the world. Therefore, easy-to-use crowdsourcing techniques should be developed in order to encourage the public to participate in heritage conservation.

Furthermore, ground level acquisition cannot provide comprehensive coverage of tall buildings and structures, hence a complementary imaging platform such as UAV is sought. Especially in the case of larger sites, aerial platforms can be significantly more efficient. A hierarchical image acquisition and reconstruction strategy will be tested in future work.

\footnotetext{
${ }^{5} \mathrm{http}: / / \mathrm{www} . c l o u d c o m p a r e . o r g$
} 


\section{ACKNOWLEDGEMENTS}

The authors would like to acknowledge the UK Engineering and Physical Sciences Research Council (EPSRC) for the financial support of the 'Disaster Risk Reduction of Heritage Structures in Nepal' project', and UNESCO Kathmandu Office, NSET, KVPT Department of Archaeology, and other local partners in Nepal for their tremendous support.

\section{REFERENCES}

Acharya, K.P. and Pradhananga, S., 2013. Review of the integrated management plan of Kathmandu Valley World Heritage property. In International Symposium on Revisiting Kathmandu, Safegurding Living Urban Heritage. pp. 127-132.

Boochs, F., Heinz, G., Huxhagen, U. and Müller, H., 2007. Lowcost image based system for nontechnical experts in cultural heritage documentation and analysis. In International Archives of the Photogrammetry, Remote Sensing and Spatial Information Sciences. pp. 165-170.

Bui, T.T., Limam, A., Sarhosis, V. and Hjiaj, M., 2017. Discrete element modelling of the in-plane and out-of-plane behaviour of masonry walls constructed with dry joints. Engineering Structures, 136, pp.277-294.

Eisenbeiss, H., 2004. A mini unmanned aerial vehicle (UAV): system overview and image acquisition. International Archives of the Photogrammetry, Remote Sensing and Spatial Information Sciences, 36(5/W1).

El-Hakim, S.F., Beraldin, J.-A., Picard, M. and Godin, G., 2004. Detailed 3D reconstruction of large-scale heritage sites with integrated techniques. IEEE Computer Graphics and Applications, 24(3), pp.21-29.

Giamundo, V., Sarhosis, V., Lignola, G.P., Sheng, Y. and Manfredi, G., 2014. Evaluation of different computational modelling strategies for the analysis of low strength masonry structures. Engineering Structures, 73(73), pp.160-169.

Guarnieri, A., Vettore, A., El-Hakim, S. and Gonzo, L., 2004. Digital photogrammetry and laser scanning in cultural heritage survey. The International Archives of the Photogrammetry, Remote Sensing and Spatial Information Sciences, 35, p.B5.

Guidi, G., Remondino, F., Morlando, G., Del Mastio, A., Uccheddu, F. and Pelagotti, A., 2007. Performances evaluation of a low cost active sensor for cultural heritage documentation. In Conference on Optical 3-D Measurement Techniques. pp. 5969.

Hanke, K. and Grussenmeyer, P., 2002. Architectural Photogrammetry: basic theory, procedures, tools. In ISPRS Commission. pp. 1-2.

Jigyasu, R., 2013. International initiatives for disaster risk management of cultural heritage. In International Symposium on Revisiting Kathmandu, Safegurding Living Urban Heritage. pp. 277-282.

Kersten, T.P. and Lindstaedt, M., 2012. Image-Based Low-Cost Systems for Automatic 3D Recording and Modelling of Archaeological Finds and Objects. In International Conference on Progress in Cultural Heritage Preservation. pp. 1-10.
Maskey, P.N., 2013. Disaster risk of culture heritage sites of the Kathmandu Valley. In International Symposium on Revisiting Kathmandu, Safegurding Living Urban Heritage. pp. 283-290.

Mills, J. and Barber, D., 2004. Geomatics Techniques for Structural Surveying. Journal of Surveying Engineering, 130(2), pp.56-64.

Mills, J.P., Peirson, G.C., Newton, I. and Bryan, P.G., 2000. Photogrammetric investigation into the suitability of desktop image measurement software for architectural recording. In International Archives of Photogrammetry and Remote Sensing. pp. 525-532.

Nex, F. and Remondino, F., 2014. UAV for 3D mapping applications: a review. Applied Geomatics, 6(1), pp.1-15.

Pavlidis, G., Koutsoudis, A., Arnaoutoglou, F., Tsioukas, V. and Chamzas, C., 2007. Methods for 3D digitization of cultural heritage. Journal of cultural heritage, 8(1), pp.93-98.

Remondino, F., 2011. Heritage recording and 3D modeling with photogrammetry and 3D scanning. Remote Sensing, 3(6), pp.1104-1138.

Remondino, F. and El-Hakim, S., 2006. Image-based 3D modelling: a review. The Photogrammetric Record, 21(115), pp.269-291.

Remondino, F. and Menna, F., 2008. Image-based surface measurement for close-range heritage documentation. International Archives of the Photogrammetry, Remote Sensing and Spatial Information Sciences, XXXVII(B5-1), pp.199-206.

Remondino, F. and Rizzi, A., 2010. Reality-based 3D documentation of natural and cultural heritage sites - techniques, problems, and examples. Applied Geomatics, 2(3), pp.85-100.

Reu, J.D., Plets, G., Verhoeven, G., Smedt, P.D., Bats, M., Cherretté, B., Maeyer, W.D., Deconynck, J., Herremans, D. and Laloo, P., 2013. Towards a three-dimensional cost-effective registration of the archaeological heritage. Journal of Archaeological Science, 40(2), pp.1108-1121.

Santagati, C., Inzerillo, L. and Di Paola, F., 2013. Image-based modeling techniques for architectural heritage 3D digitalization: limits and potentialities. The International Archives of the Photogrammetry, Remote Sensing and Spatial Information Sciences, XL(5), pp.555-560.

Sarhosis, V., Asteris, P., Wang, T., Hu, W. and Han, Y., 2016. On the stability of ancient colonnades under static and dynamic conditions. Bulletin of Earthquake Engineering, pp.1-22.

Shakya, L., Takada, M., Morishige, S. and Okubo, T., 2013. Community involvement in management of communal space in Patan Historic City. In International Symposium on Revisiting Kathmandu, Safegurding Living Urban Heritage. pp. 197-206.

Tiwari, S.R., 2013. Community participation in heritage affairs. In International Symposium on Revisiting Kathmandu, Safegurding Living Urban Heritage. pp. 189-196.

Vincent, M.L., Gutierrez, M.F., Coughenour, C., Manuel, V., Bendicho, L.-M., Remondino, F. and Fritsch, D., 2015. Crowdsourcing the 3D digital reconstructions of lost cultural heritage. In IEEE Digital Heritage. pp. 171-172. 